\title{
Simplicial Brill wave initial data
}

\author{
Adrian P. Gentlef \\ Department of Mathematics and Statistics, \\ Monash University, Clayton Victoria 3168, Australia \\ and \\ Theoretical Division (T-6, MS B288), \\ Los Alamos National Laboratory, Los Alamos, NM 87545, USA
}

\begin{abstract}
Regge calculus is used to construct initial data for vacuum axisymmetric Brill waves at a moment of time symmetry. We argue that only a tetrahedral lattice can successfully reproduce the continuum solution, and develop a simplicial axisymmetric lattice based on the co-ordinate structure of the continuum metric. This is used to construct initial data for Brill waves in an otherwise flat spacetime, and for the distorted black hole spacetime of Bernstein. These initial data sets are shown to be second order accurate approximations to the corresponding continuum solutions.
\end{abstract}

PACS numbers:

\section{Introduction}

Since it's inception in 1961, Regge calculus [1] has been extensively studied in highly symmetric spacetimes, for which corresponding exact solutions of Einstein's equations are often available. A complete review and bibliography of this early work is provided by Williams and Tuckey [2].

Following the realization that a fully decoupled, parallelizable, three-plus-one dimensional evolution scheme occurs naturally within Regge calculus 3, 4, 5], this simplicial approach to gravity is now on the verge of tackling physically interesting and dynamic problems. The first tentative step along this path was recently completed, with the successful application of simplicial Regge calculus to the Kasner spacetime in $(3+1)$-dimensions [6].

A vital precursor to the evolution problem in any general relativistic simulation is the construction of consistent initial data. Gentle and Miller [6] present a general prescription for the calculation of two-surface initial data for the Regge lattice, although their approach has only been applied to the Kasner cosmology. As a prelude to the construction of simplicial initial data for complex spacetimes, in this paper we consider the restricted case of vacuum, axisymmetric initial data at a moment of time symmetry. This is the first step towards our goal, and provides a benchmark against which future, fully four-dimensional, initial data may be compared.

We construct vacuum initial data for the pure Brill wave spacetime [7, and for Brill waves in a black hole spacetime - the "distorted black holes" first considered by Bernstein [8]. Standard finite-difference techniques have been used to study pure

$\dagger$ Email address adrian@lanl.gov. 
Brill wave spacetimes by Eppley [9], Miyama [10], Holz et al [11] and Alcubierre et al 12]. The axisymmetric Brill wave plus black hole spacetime has been extensively investigated by Bernstein [8, and more recently, a generalization to $(3+1)$-dimensions has been considered by Camarda [13].

Dubal 14] constructed pure Brill wave initial data using Regge calculus, with a lattice based on prisms rather than simplices. A similar approach was taken in an earlier version of the current work [15]. We will consider the advantages and disadvantages of using a prism-based lattice, and argue that the prism-based construction cannot, in general, satisfactorily reproduce the continuum solution, even in axisymmetry.

We begin in section 2 with a short survey of the important results from the continuum theory. In section 3 we discuss the initial value problem at a moment of time symmetry in Regge calculus, and then proceed in section 4 to examine an axisymmetric lattice built from prisms. In section 5 we turn to a fully simplicial lattice, and finally, in section 6, we analyze the convergence of the simplicial initial data to the continuum solution.

\section{The time symmetric initial value problem}

The vacuum constraint equations of General Relativity may be written in the form (see, for example, Misner, Thorne and Wheeler [16])

$$
\begin{aligned}
& R+(\operatorname{tr} K)^{2}+K_{a b} K^{a b}=0 \\
& \nabla_{b}\left(K^{a b}-\gamma^{a b} \operatorname{tr} K\right)=0
\end{aligned}
$$

where $R_{b c d}^{a}$ is the intrinsic curvature tensor on the spacelike hypersurface, and $R$ is the Ricci scalar. The extrinsic curvature $K_{a b}$ determines the embedding of this slice in the spacetime. At a moment of time symmetry $K_{a b}=0$, and the constraints reduce to vanishing of the scalar three-curvature:

$$
R=0 \text {. }
$$

The standard approach to the solution of this equation involves a conformal decomposition of the three-metric, such that

$$
\gamma_{a b}=\psi^{4} \bar{\gamma}_{a b}
$$

which recasts equation (3) in the form

$$
\nabla^{2} \psi=\frac{1}{8} \psi \bar{R}
$$

which is linear in the conformal factor $\psi$. The base metric $\bar{\gamma}_{a b}$ is used to calculate the base scalar curvature $\bar{R}$. The base three-geometry may be freely specified, although the choice may restrict the class of possible solutions. Solving this single linear elliptic partial differential equation yields the full initial data set at a moment of time symmetry.

\subsection{Pure gravitational radiation}

In this section we introduce the axisymmetric vacuum initial value problem posed by Brill [7. Conformal decomposition using a flat base metric leads only to trivial solutions. The approach taken by Brill [7] was to introduce a metric of the form

$$
d s^{2}=\psi^{4}\left\{e^{2 q}\left(d \rho^{2}+d z^{2}\right)+\rho^{2} d \phi^{2}\right\},
$$




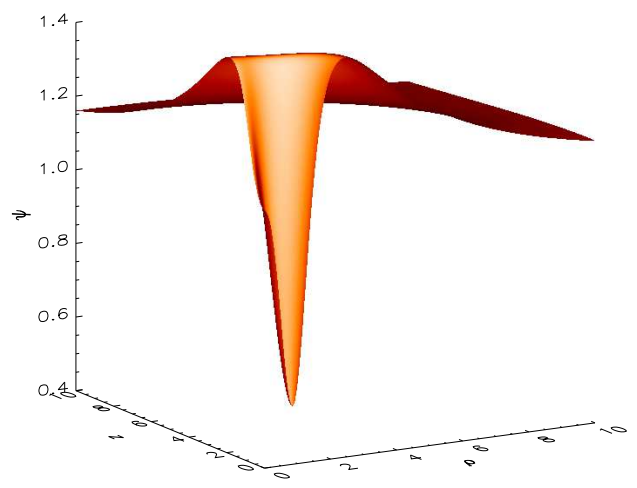

Figure 1. The conformal factor $\psi$ for Brill wave initial data, calculated on a $601 \times 601$ grid, using a centred finite difference approximation to equation (7). The Eppley form of $q(\rho, z)$ (equation (8)) was used, with wave amplitude $a=10$, and the outer boundaries were placed at $\rho=20$ and $z=20$.

where the arbitrary function $q(\rho, z)$ can be considered the distribution of gravitational wave amplitude [17]. Brill showed that $q(\rho, z)$ must satisfy the boundary conditions

$$
q(0, z)=0, \quad q_{\rho}(0, z)=0, \quad \text { and } \quad q_{z}(\rho, 0)=0,
$$

together with the condition that $q=\mathcal{O}\left(r^{-2}\right)$ as $r \rightarrow \infty$, to ensure that the hypersurface has an asymptotically well defined mass. With this choice of background metric, the Hamiltonian constraint (5) takes the form

$$
\nabla^{2} \psi=-\frac{\psi}{4}\left(\frac{\partial^{2} q}{\partial \rho^{2}}+\frac{\partial^{2} q}{\partial z^{2}}\right)
$$

which is solved for $\psi(\rho, z)$ once $q(\rho, z)$ is given.

To allow comparison with Eppley [9], we choose $q(\rho, z)$ to be of the form

$$
q=\frac{a \rho^{2}}{1+r^{n}} \quad \text { where } \quad r^{2}=\rho^{2}+z^{2},
$$

and the boundary conditions on $q$ imply that $n \geq 4$. In the remainder of this paper we set $n=5$. The single remaining parameter, the wave amplitude $a$, is arbitrary.

To obtain an axisymmetric, asymptotically flat solution which is reflection symmetric on the $z=0$ axis, we use boundary conditions on $\psi$ of the form

$$
\frac{\partial \psi}{\partial \rho}(0, z)=0, \quad \frac{\partial \psi}{\partial z}(\rho, 0)=0
$$

together with a Robin outer boundary condition,

$$
\frac{\partial \psi}{\partial r}=\frac{1-\psi}{r} \quad \text { as } \quad r \rightarrow \infty
$$

Figure 1 1 a shows the solution of equation (7) obtained using a centred finite difference approximation to both the equation and boundary conditions. The solution was calculated on a $601 \times 601$ grid with a Brill wave amplitude $a=10$. 


\subsection{Distorted black hole initial data}

We now turn to the Brill wave plus black hole spacetime, investigated in great detail by Bernstein [8]. As before, a perturbation is introduced onto the background metric, and the conformal factor is calculated from the single initial value equation (5). Note that we solve the full initial value problem for the wave and black hole together. This is not a perturbation solution.

Mirroring the pure Brill wave spacetime of section 2.1, we write the physical metric on the initial surface in the form

$$
d s^{2}=\psi^{4}\left\{\mathrm{e}^{2 q}\left(d \eta^{2}+d \theta^{2}\right)+\sin ^{2} \theta d \phi^{2}\right\},
$$

where the exponential radius co-ordinate $\eta$ is defined from $\rho=m \exp (\eta) / 2$. The "mass" $m$ is that of the black hole alone, obtained by setting $q(\eta, \theta)=0$. In $(\eta, \theta)$ co-ordinates, the isolated black hole solution takes the form

$$
\psi_{b h}=\sqrt{2 m} \cosh (\eta / 2) .
$$

The Hamiltonian constraint (5) evaluated on the black hole plus Brill wave metric, equation (11), yields the linear constraint

$$
\nabla^{2} \psi=-\frac{\psi}{4}\left(\frac{\partial^{2} q}{\partial \eta^{2}}+\frac{\partial^{2} q}{\partial \theta^{2}}-1\right)
$$

which is again solved for the conformal factor $\psi$ once the perturbation $q(\eta, \theta)$ is given. We choose boundary conditions of the form

$$
\frac{\partial \psi}{\partial \eta}(0, \theta)=0, \quad \frac{\partial \psi}{\partial \theta}(\eta, 0)=0, \quad \frac{\partial \psi}{\partial \theta}\left(\eta, \frac{\pi}{2}\right)=0,
$$

on the "inner" boundaries, where $\eta=0$ corresponds to an Einstein-Rosen bridge at $r=m / 2$. These represent reflection symmetry on the $\theta=0, \theta=\pi / 2$ and $\eta=0$ boundaries. The outer boundary condition is again applied using the Robin approach, adapted to the exponential radial co-ordinate $\eta$. Examining the asymptotic form of the black hole solution yields the condition

$$
\frac{\partial \psi}{\partial \eta}+\frac{\psi}{2}=\sqrt{\frac{m}{2}} e^{\eta / 2},
$$

on the outer boundary.

As a test of the axisymmetric code, we solve equation (13) using a second-order finite difference approximation with $q(\eta, \theta)=0$, together with the boundary conditions above. We expect that the solution $\psi_{i j}$ will converge as the second power of the grid spacing to the analytic black hole solution, given by equation (12). We have performed such a calculation, and find that the axisymmetric numerical solution does indeed converge as the second power of the grid spacing to the black hole solution. This provides some confidence in the the continuum code, which is vital since it will be used later to benchmark the Regge calculations.

Returning to the construction of distorted black hole initial data, we follow Bernstein and choose $q(\eta, \theta)$ to be of the form

$$
q=a \sin ^{2} \theta\left\{e^{-g_{+}^{2}}+e^{-g_{-}^{2}}\right\}
$$

with $g_{ \pm}=(\eta \pm b) / \omega$, which contains the set of free parameters $(a, b, \omega)$.

The solution of the Hamiltonian constraint (13) is shown in figure 2 for several illustrative choices of the free parameters. The figure shows the ratio of the conformal 


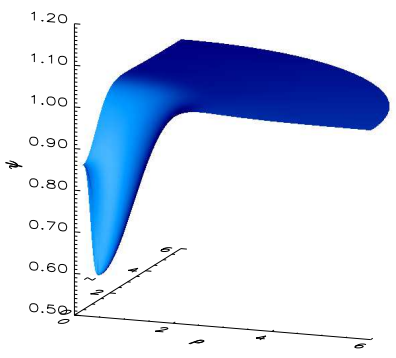

(a)

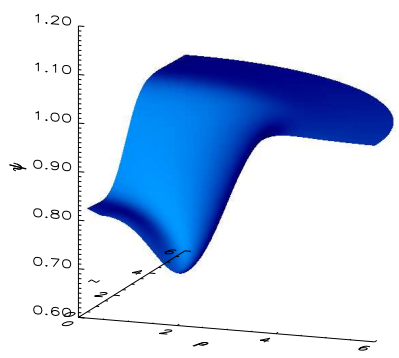

(c)

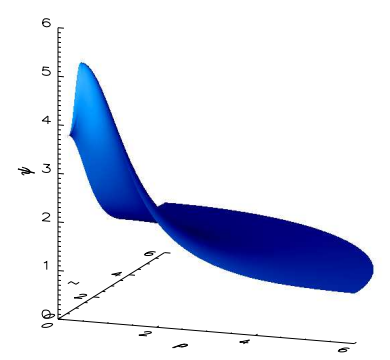

(b)

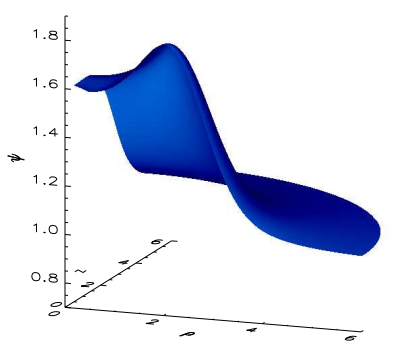

(d)

Figure 2. The continuum solution for the black hole plus Brill wave spacetime. The conformal factor is plotted as a ratio of the black hole solution $\left(\psi / \psi_{b h}\right)$, with the results calculated on a $601 \times 601$ grid with $m=1$. An artificial Cartesian projection is used, and this is defined in the text. The choice of parameters are (a) $(1,0,1),($ b) $(-1,0,1),($ c) $(1,2,1)$ and $(d)(-1,2,1)$.

factor to the black hole solution, $\psi / \psi_{b h}$. The outer boundary was placed at $\eta=6$, and the results are plotted on a pseudo-Cartesian grid defined by

$$
\begin{aligned}
& x=\left(\eta+\eta_{0}\right) \sin \theta \\
& y=\left(\eta+\eta_{0}\right) \cos \theta
\end{aligned}
$$

where $\eta_{0}=\frac{1}{2}$ is used to display an artificial throat in $(\eta, \theta)$ coordinates. These results compare well with the calculations of Bernstein [8], who also used a second order finite-difference approximation to the Hamiltonian constraint.

\section{The Regge initial value problem at a moment of time symmetry}

We found in section 2 that the continuum initial value problem at a moment of time symmetry reduces to the vanishing of the scalar three-curvature intrinsic to the hypersurface. By using this knowledge, the full two-slice Regge initial value problem can be reduced to a purely three-dimensional calculation.

The Regge equivalent of the scalar curvature for a three dimensional hypersurface has been given by Wheeler [17. The Regge equivalent of $R=0$ is

$$
\sum_{b} L_{a b} \varepsilon_{a b}=0
$$


where $L_{a b}$ is the edge joining vertex $a$ to vertex $b$, and $\varepsilon_{a b}$ is the deficit angle (curvature) about $L_{a b}$. The summation is over all edges $L_{a b}$ which meet at vertex $a$.

In order to solve this restricted initial value problem, we mirror the continuum formulation and perform a conformal decomposition. This reduces the problem to the solution of the single Regge-Hamiltonian constraint, equation (17), for the simplicial conformal factor. The conformal factor is defined on the vertices of the lattice, and we perform the decomposition on the lattice edge lengths, since they correspond closely to the continuum metric. For the edge $L_{a b}$ connecting vertices $a$ and $b$, the decomposition takes the form

$$
L_{a b}=\psi_{a b}^{2} \bar{L}_{a b}
$$

where $\bar{L}_{a b}$ is the freely chosen base edge length. Since the conformal factor is defined on the vertices, we use the second order accurate expression

$$
\psi_{a b}=\frac{1}{2}\left(\psi_{a}+\psi_{b}\right)
$$

to define the conformal factor $\psi_{a b}$ acting on the edge $L_{a b}$, where $\psi_{a}$ is the conformal factor at vertex $a$.

So far we have considered only the restricted case of initial data at a moment of time symmetry - a purely three dimensional problem. However, it is possible to use the solution to equation (17) to obtain full axisymmetric two-surface simplicial initial data.

After solving equation (17), we have a set of physical edges $\left\{L_{a b}\right\}$, which fix the geometry on a simplicial three-surface. Using the time-symmetry condition, we can construct two-slice initial data by creating two identical three-dimensional lattices using these edges, and then filling in the intervening four-volume with four-simplices. This involves introducing a timelike edge to join equivalent vertices in the two threegeometries, together with a "brace" edge which stretches from one surface to the next, with one such brace lying above each edge in the lower surface. This constructs a set of four-simplices between the two surfaces. Care must be taken in the choice of these braces if a particular lattice structure is desired. In particular, if we require a lattice amenable to the Sorkin evolution scheme [5].

Assuming symmetry about the centre of the intervening volume, the squared lengths $b_{a b}^{2}$ of the brace edges stretching from one surface to the next are

$$
b_{a^{+b}}^{2}=L_{a b}^{2}+\tau_{a^{+} a}^{2}
$$

where vertex $a^{+}$lies on the upper surface, and $\tau_{a^{+a}}^{2}=\tau^{2}<0$ is the proper time between the two slices, measured along the timelike edges connecting equivalent vertices. All such timelike edges have the same length, and we require that $-\tau^{2}<<$ $\min \left\{L_{a b}^{2}\right\}$ in order to obtain a reasonable approximation to the time-symmetry condition. This completes the solution of the full two-surface initial value problem at a moment of time symmetry.

\section{A lattice built from prisms?}

So far the discussion of Regge calculus has been general, without the need to restrict attention to one particular lattice. Before we can construct a solution numerically, we must choose a particular form of lattice to work with. In this section we discuss 


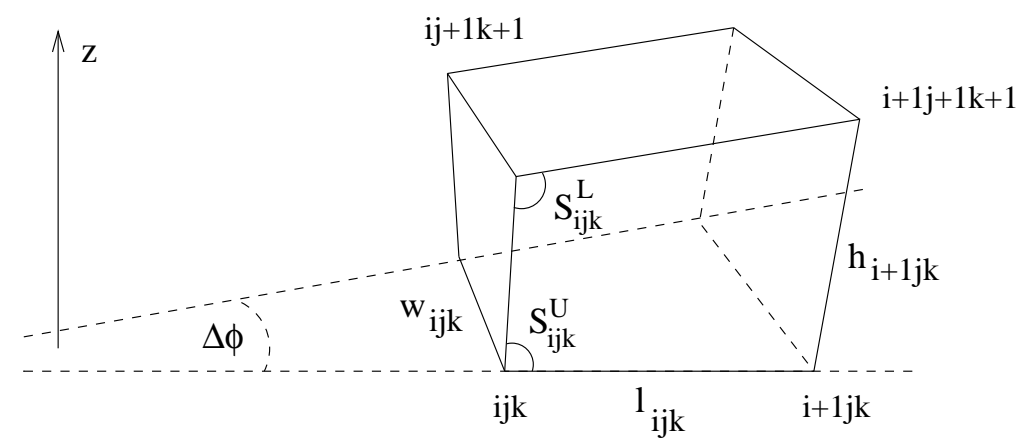

Figure 3. A section of the axisymmetric lattice used by Dubal [14]. The relation to the global polar co-ordinate system is shown, together with the angles used to specify the extra degree of freedom.

the application of a prism-based lattice to the axisymmetric initial value problem in Regge calculus at a moment of time symmetry.

Much previous work in Regge calculus has been based on simple lattice structures, often built from prisms. Typical examples include the work of Lewis [18], where several $T^{3} \times R$ cosmological models were approximated with a hypercubic lattice, and Collins and Williams 19], where tetrahedra were used to build each three-geometry, but the world-tube of each tetrahedra was not fully subdivided into four-simplices.

The principle advantages of this prism-based approach are the ability to closely model the symmetries of the spacetime of interest, and the computational simplicity when compared with a simplicial lattice. Since there are less edges in the lattice, there are less hinges about which curvature is concentrated, and hence less calculations are required to obtain the deficit angles within the lattice.

It is largely for these reasons that the prism approach has dominated much previous work in Regge calculus. The major drawback of a prism-based lattice is that even when all edges have been specified, the lattice is still not rigid; additional constraints must be provided to constrain the space of possible edge lengths. In the presence of high symmetry, it is possible that natural restrictions are available to specify the remaining degrees of freedom. This has been the case for most previous prism-based applications of Regge calculus.

The simplicial approach, which constructs three-manifolds from tetrahedral and four-manifolds from four-simplices, is more natural if one wishes to model a complex spacetime. In a simplicial lattice, once all edge lengths have been specified, the geometry is uniquely determined. Noting that a simplicial lattice may be obtained by subdivision of a prism-based lattice, some of the advantages of the prism construction may be carried over to the simplicial lattice.

For the particular case of axisymmetric non-rotating Brill waves, the symmetry suggests that a prism-based lattice may be convenient. Indeed, Dubal [14] approximated the pure Brill wave spacetime at a moment of time symmetry with a lattice constructed from the co-ordinate blocks of the continuum, a typical element of which is shown in figure 3. The desired axisymmetry is built into the lattice by demanding that there is no variation along the $\phi$-axis. The limit as $\Delta \phi$ tends to zero is then taken to obtain a purely axisymmetric lattice.

Dubal fixed the remaining degrees of freedom in the prisms by introducing several 
angles on each $\rho-z$ face, as shown in figure 3, and specifying them in terms of the surrounding edges. The clever assignment chosen by Dubal allowed him to construct Regge solutions in reasonable agreement with the continuum solution, at least for low amplitude Brill waves. Dubal noted several limitations to his approach, including the relatively poor Regge mass estimates for "large amplitude" $\left(a \approx \frac{1}{2}\right)$ Brill waves. Another indication of problems was the low convergence rate of the prism-based Regge solution to the continuum. As Dubal notes [14], these problems arise because of the approximation made in specifying the angles within each prism. To obtain a better solution, an improved relation between the angles and prism edges is required. We argue that the best solution is to abandon the prisms altogether, and introduce a tetrahedral lattice.

Once the axisymmetric limit $(\Delta \phi \rightarrow 0)$ has been taken, the major difference between a prism-based and a tetrahedral lattice is the absence of a diagonal edge on the $\rho z$ faces. Each such face in a prism-based lattice is required, by construction, to be flat. However, the pure Brill wave metric (6) allows fluctuations across faces in the $\rho-z$-plane, through both the conformal factor $\psi(\rho, z)$ and the form function $q(\rho, z)$. A prism-based lattice is unable to capture such variations.

The most natural solution to this problem is to abandon the prism-based lattice for one constructed from simplices. In the remainder of this paper, we shall follow just such a path, and show that a tetrahedral lattice resolves all of the problems encountered by Dubal.

\section{A tetrahedral three-geometry}

In this section we construct an axisymmetric, shear-free simplicial three-geometry, and use it to build time symmetric initial data using Regge calculus.

The simplicial lattice may be obtained by subdividing the basic prism shown in figure 3. Each block is divided into six tetrahedra, introducing three face diagonals and one body diagonal per vertex. The result is shown in figure 1 . The body diagonal within the prism is denoted $b_{i j k}$, whilst face diagonals spanning the $\rho$ and $\phi$-axes are written $d_{i j k}^{\rho \phi}$, and so forth.

To obtain an axisymmetric approximation in the style of the preceding section, we take the limit as the prism is collapsed along the $\phi$-axis. This is a more complicated procedure than in the previous case [14], since we must now deal with the limiting form of the extra edges introduced in the simplectic approach, together with their associated deficit angles.

To aid in constructing the lattice, we consider the "prototype" axisymmetric metric

$$
d s^{2}=d \rho^{2}+d z^{2}+\rho^{2} d \phi^{2} .
$$

This non-physical metric is useful to frame our discussion of the axisymmetric lattice, and later we will map from this "prototype" to metrics of physical interest. Figure 1 shows the subdivision of a co-ordinate block, such that the $l, h$ and $w$ edges are locally aligned with the $\rho, z$ and $\phi$-axes respectively. We enforce axisymmetry by explicitly setting

$$
w_{i j k}=r_{i j k} \Delta \phi,
$$

and demanding that there be no variation in the edges along the $\phi$-axis. The redundant $j$ index is neglected in the remainder of this paper. 


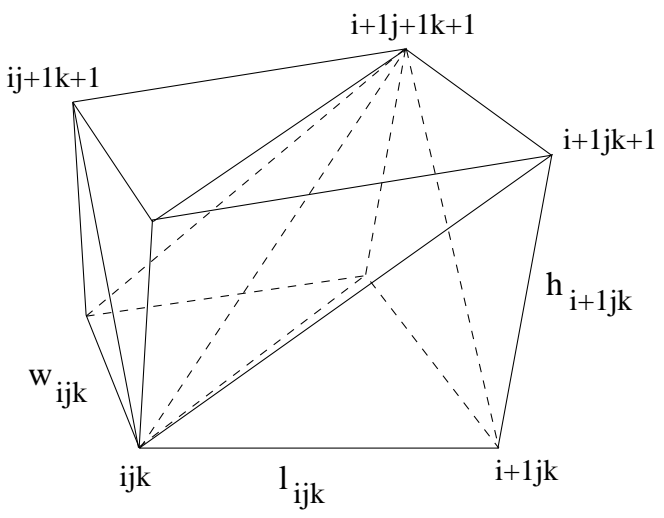

Figure 4. The rectangular prism shown in figure 3 subdivided into six tetrahedra. This involves adding a diagonal brace to each face of the prism, together with a body diagonal.

Consider the behaviour of the lattice in the limit as $\Delta \phi$ approaches zero. For a single triangle with edge lengths $a, b$ and $r \Delta \phi$, in the limit as $\Delta \phi \rightarrow 0$ we require that $a=b$. In general, the manner in which the edges $a$ and $b$ approach this limit is not uniquely defined. However, as we shall see, the assumption of axisymmetry uniquely determines the leading order terms in the expansions about $\Delta \phi=0$.

Assuming that the lattice is both axisymmetric and shear-free, the $\rho-\phi$ and $z-\phi$ faces of the block must be flat as $\Delta \phi$ approaches zero, since any deviation from flatness introduces an asymmetry across the prism. This allows us to write an expansion in $\Delta \phi$ for the diagonal edges on these two faces. For the diagonal edge within the $\rho-\phi$ face we have

$$
\left\{d_{i k}^{\rho \phi}\right\}^{2}=l_{i k}^{2}+r_{i k} r_{i+1 k} \Delta \phi^{2},
$$

and similarly, the diagonals which span $z \phi$ faces of the prism may be expanded as

$$
\left\{d_{i k}^{z \phi}\right\}^{2}=h_{i k}^{2}+r_{i k} r_{i k+1} \Delta \phi^{2}
$$

in the limit as $\Delta \phi$ tends to zero.

The only remaining edge which spans the $y$-axis is the body diagonal, $b_{i k}$. The shear-free and axisymmetric nature of the lattice also implies that the plane formed by the four vertices $(i, j, k),(i, j+1, k),(i+1, j, k+1)$ and $(i+1, j+1, k+1)$ is flat as $\Delta \phi$ tends to zero, and so the body diagonal may be expanded as

$$
b_{i k}^{2}=\left(d_{i k}^{\rho z}\right)^{2}+r_{i k} r_{i+1 k+1} \Delta \phi^{2},
$$

to leading order in $\Delta \phi$.

We now turn to the calculation of the deficit angles. Consider a tetrahedron consisting of the vertices $(0,1,2,3)$, with corresponding edges $L_{a b}$, where $a$ and $b$ are vertices of the tetrahedron. The dihedral angle $\theta_{01}^{0123}$ about the edge joining vertices 0 and 1 within the tetrahedron 0123 is

$$
\begin{aligned}
\cos \theta_{01}^{0123}=\frac{-1}{16 A_{012} A_{013}}\{ & L_{01}^{4}+2 L_{01}^{2} L_{23}^{2}-L_{01}^{2} L_{02}^{2}-L_{01}^{2} L_{03}^{2} \\
& -L_{01}^{2} L_{12}^{2}-L_{01}^{2} L_{13}^{2}+L_{02}^{2} L_{03}^{2}+L_{12}^{2} L_{13}^{2} \\
& \left.-L_{02}^{2} L_{13}^{2}-L_{03}^{2} L_{12}^{2}\right\}
\end{aligned}
$$


where $A_{012}$ and $A_{013}$ are the areas of the triangular faces 012 and 013 respectively, given by

$$
16 A_{a b c}^{2}=-L_{a b}^{4}-L_{a c}^{4}-L_{b c}^{4}+2 L_{a b}^{2} L_{a c}^{2}+2 L_{a b}^{2} L_{b c}^{2}+2 L_{b c}^{2} L_{a c}^{2} .
$$

Equations (24) and (25) are used to calculate all dihedral angles within the prism of figure 1 . Consider, for example, the deficit angle about the edge $l_{i k}$. There are six tetrahedra hinging on this edge, so we must calculate the dihedral angle about $l_{i k}$ in each of these tetrahedra, after which a power series expansion is taken about $\Delta \phi=0$, using equations (21) - (23). Retaining only the leading power in $\Delta \phi$, the defect about $l_{i k}$ is

$$
\begin{aligned}
\epsilon\left(l_{i k}\right)= & {\left[\frac{l_{i k}^{2}\left(r_{i k}+r_{i+1 k}-2 r_{i+1 k+1}\right)+\Delta_{i} r_{i k}\left(d_{i k}^{2}-h_{i+1 k}^{2}\right)}{4 l_{i k} A_{i k}^{B}}\right.} \\
& \left.+\frac{l_{i k}^{2}\left(r_{i k}+r_{i+1 k}-2 r_{i k-1}\right)-\Delta_{i} r_{i k}\left(d_{i k-1}^{2}-h_{i k-1}^{2}\right)}{4 l_{i k} A_{i k-1}^{T}}\right] \Delta \phi,
\end{aligned}
$$

where the two triangular areas constituting a $\rho-z$ face are given by

$$
\begin{aligned}
A_{i k}^{B} & =\frac{1}{4} \sqrt{2 l_{i k}^{2} d_{i k}^{2}+2 l_{i k}^{2} h_{i+1 k}^{2}+2 h_{i+1 k}^{2} d_{i k}^{2}-d_{i k}^{4}-l_{i k}^{4}-h_{i+1 k}^{2}} \\
A_{i k}^{T} & =\frac{1}{4} \sqrt{2 l_{i k+1}^{2} d_{i k}^{2}+2 l_{i k+1}^{2} h_{i k}^{2}+2 h_{i k}^{2} d_{i k}^{2}-d_{i k}^{4}-l_{i k+1}^{4}-h_{i k}^{2}},
\end{aligned}
$$

with $\Delta_{i} r_{i k}=r_{i+1 k}-r_{i k}$.

A similar procedure applied to the six tetrahedra about the edge $r_{i k} \Delta \phi$ yields the deficit angle

$$
\begin{aligned}
\epsilon\left(r_{i k} \Delta \phi\right)=2 \pi & -\cos ^{-1}\left(\frac{h_{i k}^{2}+d_{i k}^{2}-l_{i k+1}^{2}}{2 d_{i k} h_{i k}}\right) \\
& -\cos ^{-1}\left(\frac{h_{i k}^{2}+l_{i-1 k}^{2}-d_{i-1 k}^{2}}{2 l_{i-1 k} h_{i k}}\right) \\
& -\cos ^{-1}\left(\frac{l_{i k}^{2}+d_{i k}^{2}-h_{i+1 k}^{2}}{2 d_{i k} l_{i k}}\right) \\
& -\cos ^{-1}\left(\frac{h_{i k-1}^{2}+l_{i k}^{2}-d_{i k-1}^{2}}{2 l_{i k} h_{i k-1}}\right) \\
& -\cos ^{-1}\left(\frac{h_{i k-1}^{2}+d_{i-1 k-1}^{2}-l_{i-1 k-1}^{2}}{2 d_{i-1 k-1} h_{i k-1}}\right) \\
& -\cos ^{-1}\left(\frac{l_{i-1 k}^{2}+d_{i-1 k-1}^{2}-h_{i-1 k-1}^{2}}{2 l_{i-1 k} d_{i-1 k-1}}\right),
\end{aligned}
$$

to leading order in $\Delta \phi$. The deficit angles about the vertical edge $h_{i k}$ and the remaining diagonal edge $d_{i k}^{\rho z}$ are

$$
\begin{aligned}
\epsilon\left(h_{i k}\right)= & {\left[\frac{h_{i k}^{2}\left(r_{i k}+r_{i k+1}-2 r_{i+1 k+1}\right)+\Delta_{k} r_{i k}\left(d_{i k}^{2}-l_{i k+1}^{2}\right)}{4 h_{i k} A_{i k}^{T}}\right.} \\
& \left.+\frac{h_{i k}^{2}\left(r_{i k}+r_{i k+1}-2 r_{i-1 k}\right)-\Delta_{k} r_{i k}\left(d_{i-1 k}^{2}-l_{i-1 k}^{2}\right)}{4 h_{i k} A_{i-1 k}^{B}}\right] \Delta \phi \\
\epsilon\left(d_{i k}^{\rho z}\right)= & {\left[\frac{d_{i k}^{2}\left(r_{i k}+r_{i+1 k+1}-2 r_{i k+1}\right)-\Delta_{i k} r_{i k}\left(l_{i k+1}^{2}-h_{i k}^{2}\right)}{4 d_{i k} A_{i k}^{T}}\right.}
\end{aligned}
$$




$$
\left.+\frac{d_{i k}^{2}\left(r_{i k}+r_{i+1 k+1}-2 r_{i+1 k}\right)+\Delta_{i k} r_{i k}\left(l_{i k}^{2}-h_{i+1 k}^{2}\right)}{4 d_{i k} A_{i k}^{B}}\right] \Delta \phi
$$

where the difference operator $\Delta_{i k}$ is defined by $\Delta_{i k} r_{i k}=r_{i+1 k+1}-r_{i k}$. The remaining deficit angles are simply

$$
\epsilon\left(d_{i k}^{\rho \phi}\right)=\epsilon\left(d_{i k}^{\phi z}\right)=\epsilon\left(b_{i k}\right)=0,
$$

by virtue of the axisymmetric expansions, equations (21) - (23). Note that the

deficit about the face diagonal $d_{i k}^{\rho z}$, the only face diagonal edge remaining after the limit $\Delta \phi \rightarrow 0$ has been taken, is not identically zero. The fully simplicial model, unlike the prism method described above, can have non-zero curvature about faces in the $\rho z$-plane.

Evaluating the Regge initial value equation (17) on the simplicial lattice gives

$$
\begin{aligned}
0= & l_{i k} \epsilon\left(l_{i k}\right)+l_{i-1 k} \epsilon\left(l_{i-1 k}\right) \\
& +2 r_{i k} \Delta \phi \epsilon\left(r_{i k} \Delta \phi\right)+h_{i k} \epsilon\left(h_{i k}\right)+h_{i k-1} \epsilon\left(h_{i k-1}\right) \\
& +d_{i k} \epsilon\left(d_{i k}\right)+d_{i-1 k-1} \epsilon\left(d_{i-1 k-1}\right)
\end{aligned}
$$

to leading order in $\Delta \phi$ at each vertex $(i, k)$ in the lattice. This equation may be evaluated once all edge lengths in the lattice are given, together with the definitions of the deficit angles in terms of the lattice edges given above.

\subsection{Pure Brill wave initial data}

We now consider the case of pure Brill wave initial data, as discussed in section 2.1. In order to solve the Regge initial value equation on the tetrahedral lattice, we perform a conformal decomposition, similar to the continuum approach.

The lattice is constructed to mirror the cylindrical polar co-ordinate system in which the continuum Brill wave metric (6) is written. We obtain the Regge conformal decomposition by integrating the spacelike geodesics between vertices of the lattice, and assigning the geodesic lengths directly to the lattice edges. It is sufficient to take only the leading order terms in the expansion.

To construct the Regge conformal decomposition for axisymmetric Brill waves, we must map the lattice edges from the "prototype metric", equation (19), to the Brill wave metric, equation (6). Performing this mapping yields

$$
\begin{aligned}
l_{i k} & =\left[\psi^{2} e^{q}\right]_{i+\frac{1}{2}, k} \Delta \rho_{i} \\
h_{i k} & =\left[\psi^{2} e^{q}\right]_{i, k+\frac{1}{2}} \Delta z_{k} \\
d_{i k} & =\left[\psi^{2} e^{q}\right]_{i+\frac{1}{2} k+\frac{1}{2}}\left(\Delta \rho_{i}^{2}+\Delta z_{k}^{2}\right)^{1 / 2} \\
r_{i k} \Delta \phi & =\psi_{i k}^{2} \rho_{i} \Delta \phi .
\end{aligned}
$$

The notation $H_{i+\frac{1}{2}, k}$ indicates that the quantity $H$ is centred between the $(i, k)$ and $(i+1, k)$ vertices.

The boundary conditions applied to the lattice are the same as those used to construct the continuum solution - equations (9) and (10). They are applied using a second order power series expansion into the domain. On the $\rho=0$ boundary we take

$$
\psi=C_{0}+C_{1} \rho+C_{2} \rho^{2}+O\left(\rho^{3}\right),
$$




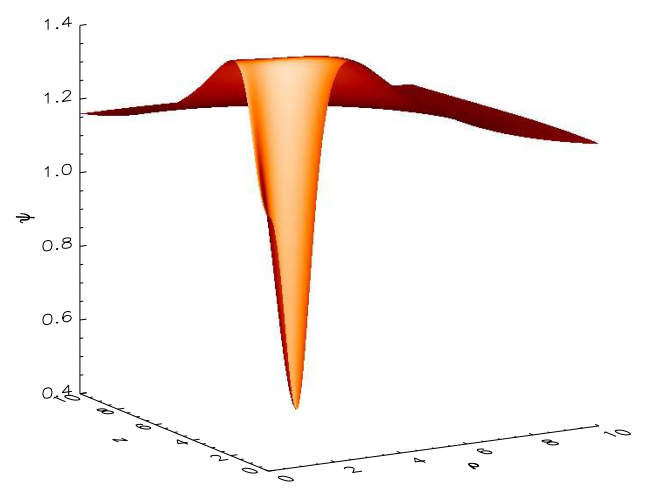

Figure 5. The conformal factor $\psi$ for a Brill wave of amplitude $a=10$ calculated using the simplicial Regge lattice. The agreement between this and the continuum solution in figure 1 is excellent. Calculations were performed using a $601 \times 601$ lattice, with the outer boundaries placed at $\rho=20$ and $z=20$.

where the $C_{i}$ are constants. Applying reflection symmetry gives $C_{1}=0$, and evaluating the expansion at $\rho=0, \Delta \rho, 2 \Delta \rho$ yields the condition

$$
\psi_{0 k}=\frac{1}{3}\left(4 \psi_{1 k}-\psi_{2 k}\right) \text {. }
$$

Similarly, the reflection boundary condition at $z=0$ takes the form

$$
\psi_{i 0}=\frac{1}{3}\left(4 \psi_{i 1}-\psi_{i 2}\right),
$$

and at the inner point $\rho=z=0$ an expansion in both $\rho$ and $z$ into the domain suggests a boundary condition of the form

$$
\psi_{00}=\psi_{01}+\psi_{10}-\psi_{11} .
$$

The outer boundary condition, equation (10), is applied to the lattice using a centred finite difference approximation to the derivative. It is first converted to pure $\rho$ or $z$ derivatives, to give

$$
\begin{aligned}
& \frac{\partial \psi}{\partial \rho}=\frac{\rho}{r}\left(\frac{1-\psi}{r}\right) \quad \text { along } \quad \rho=\rho_{\max } \\
& \frac{\partial \psi}{\partial z}=\frac{z}{r}\left(\frac{1-\psi}{r}\right) \quad \text { along } \quad z=z_{\max }
\end{aligned}
$$

and then evaluated one point in from the relevant outer boundary.

To complete the construction, the lattice spacings $\Delta \rho_{i}$ and $\Delta z_{k}$ are chosen, together with an initial guess for the conformal factor $\psi$ (taken to be $\psi=1$ ). The edge lengths on the lattice are then calculated using equations (31), again using equation (8) to specify $q(\rho, z)$. Finally, the Regge-Hamilton constraint (30) is solved at each vertex, together with the boundary conditions described above.

The Newton-Raphson method is used to linearize the system, which is then solved using a sparse storage biconjugate gradient algorithm. This process is repeated until the desired convergence criteria is reached; in our case, a fractional change in the conformal factor of less than 1 part in $10^{-12}$. Figure 5 displays the solution to equation 


\begin{tabular}{|c|c|c|c|}
\hline $\mathrm{a}$ & $M_{S}$ & $M_{C}$ & $M($ Alcubierre et al $)$ \\
\hline 1 & $4.7 \times 10^{-2}$ & $4.7 \times 10^{-2}$ & $4.8 \times 10^{-2}$ \\
2 & $1.72 \times 10^{-1}$ & $1.72 \times 10^{-1}$ & $1.74 \times 10^{-1}$ \\
5 & $8.77 \times 10^{-1}$ & $8.79 \times 10^{-1}$ & $8.83 \times 10^{-1}$ \\
10 & 3.22 & 3.22 & 3.22 \\
12 & 4.84 & 4.84 & 4.85 \\
\hline
\end{tabular}

Table 1. Mass estimates for the different initial data sets using the Eppley form function $q(\rho, z)$. The mass is calculated by examining the fall off of $\psi$ in the asymptotic region, for each data set - the simplicial Regge $\left(M_{S}\right)$ and continuum $\left(M_{C}\right)$ solutions. We also show the results of the previous calculations by Alcubierre et al 12], which are in excellent agreement with both the continuum and simplicial Regge solutions.

(30) with wave amplitude $a=10$. The solution is in excellent qualitative agreement with the continuum solution shown in figure 1. In section 6 we provide a quantitative analysis of the difference between the two solutions.

We estimate the mass of the initial data shown in figures 1 and 5 by examining the fall off of the conformal factor in the asymptotic region. Assuming that the conformal factor takes the form

$$
\psi=1+\frac{M}{2 r}+\ldots \quad \text { as } \quad r \rightarrow \infty,
$$

we perform a least squares fit of the this function to each numerical solution far from the region in which the Brill wave is concentrated. This asymptotic form for $\psi$ is guaranteed by the choice of outer boundary condition - only the value of $M$ is left undetermined. The mass estimates are shown in table 1 for various choices of Brill wave amplitude. The masses calculated for the simplicial Regge and continuum solutions agree remarkably well, and also show excellent agreement with previous calculations.

\subsection{Distorted black hole initial data via Regge calculus}

We now turn our attention to Regge initial data for Brill waves in a black hole spacetime. Since these waves may be viewed as a distortion to the Schwarzschild solution, it is natural in to represent them in spherical polar co-ordinates, as we found in section 2.2.

We approximate spherical symmetry in the lattice three-geometry by aligning the base edges of the lattice with a spherical polar co-ordinate system. This implies, for example, that the $z$-axis in figure 3 becomes the azimuthal $\theta$-axis. For convenience we also introduce an exponential radial co-ordinate $\eta$.

In the case of a single black hole with no Brill perturbations, the face $\left(h_{i k}, r_{i k}\right)$ in figure 4 lies at constant proper distance from the throat (minimal two-surface), and the edges $l_{i k}$ stretch from one such face to the next. For the remainder of this section the labels $i$ and $k$ are taken to refer to the radial and azimuthal co-ordinates, respectively. This identification of the lattice edges greatly simplifies the application of spherically symmetric boundary conditions.

The metric for black hole plus Brill wave initial data, equation (11), is used to assign the base edge lengths. Integrating the geodesics between the vertices on each edge, and retaining only leading order terms, yields a simplicial conformal 


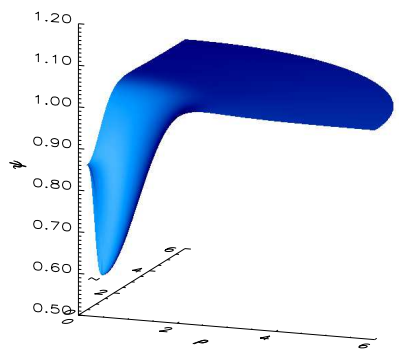

(a)

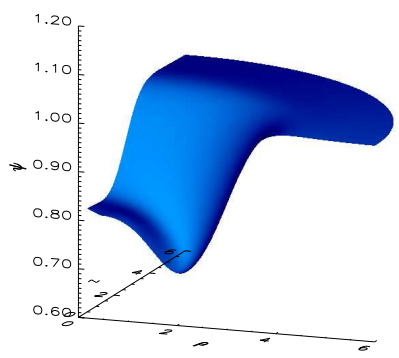

(c)

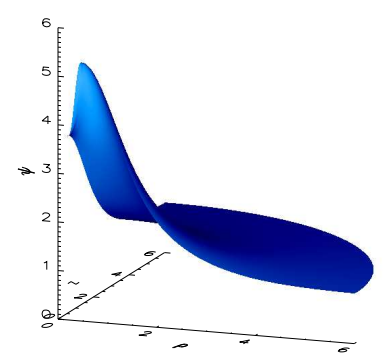

(b)

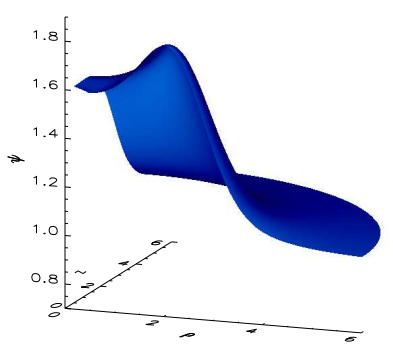

(d)

Figure 6. The conformal factor for distorted black hole initial data, shown as a ratio of the black hole conformal factor $\left(\psi / \psi_{b h}\right)$. The calculations were performed using the simplicial Regge lattice with $601 \times 601$ vertices, with the same choice of parameters as figure 22 (a) $(1,0,1),(b)(-1,0,1),(c)(1,2,1)$ and (d) $(-1,2,1)$.

decomposition of the form

$$
\begin{aligned}
l_{i k} & =\left[\psi^{2} e^{q}\right]_{i+\frac{1}{2}, k} \Delta \eta_{i} \\
h_{i k} & =\left[\psi^{2} e^{q}\right]_{i, k+\frac{1}{2}} \Delta \theta_{k} \\
d_{i k} & =\left[\psi^{2} e^{q}\right]_{i+\frac{1}{2} k+\frac{1}{2}}\left(\Delta \eta_{i}^{2}+\Delta \theta_{k}^{2}\right)^{1 / 2} \\
r_{i k} \Delta \phi & =\psi_{i k}^{2} \sin \theta_{k} \Delta \phi,
\end{aligned}
$$

where we have mapped the lattice edges from the "prototype" metric, equation (19), to the distorted black hole metric.

The inner boundary conditions are obtained as before, by combining the continuum inner boundary condition (14) with a power series expansion into the domain. The outer boundary uses a second order finite difference approximation to the Robin condition, equation (15). The inner $\eta=0$ boundary condition is

$$
\psi_{0 k}=\frac{1}{3}\left(4 \psi_{1 k}-\psi_{2 k}\right),
$$

and similarly, the reflection conditions on at $\theta=0$ and $\theta=\pi / 2$ are

$$
\psi_{i 0}=\frac{1}{3}\left(4 \psi_{i 1}-\psi_{i 2}\right)
$$




$$
\psi_{i n_{\theta}}=\frac{1}{3}\left(4 \psi_{i n_{\theta}-1}-\psi_{i n_{\theta}-2}\right) .
$$

These expansions are combined to give boundary conditions at the two "corner points" along $\eta=0$,

$$
\begin{aligned}
& \psi_{00}=\psi_{01}+\psi_{10}-\psi_{11} \\
& \psi_{0 n_{\theta}}=\psi_{0 n_{\theta}-1}+\psi_{1 n_{\theta}}-\psi_{1 n_{\theta}-1}
\end{aligned}
$$

These boundary conditions, together with the assignment of edge lengths given by equations (38), allow us to calculate and solve the initial value equation (30) as before, except that we now take the pure black hole solution (12) as the initial guess. In the following calculations we use Bernstein's [8] choice of $q(\eta, \theta)$, equation (16), together with a choice of the free parameters $(a, b, \omega)$ and the black hole mass $m$.

The solution is shown in figure 6, with the same set of parameter choices as figure 2. We again find excellent qualitative agreement between the continuum solution and the simplicial Regge initial data. In the next section we will attempt to make the comparison more rigorous.

\section{Convergence to the continuum}

We have seen in the preceding sections that the simplicial Regge solutions show reasonable agreement with solutions of the continuum equations. We now make this comparison more quantitative.

We have at hand two different numerical solutions for a given physical system and choice of the various parameters; (i) the finite-difference solution to the continuum equation (13), and (ii) the solution of the Regge initial value equation (30). Since an exact solution is not available for comparison, the convergence analysis must be based solely on these equations and corresponding solutions.

Consider the finite-difference solution $\psi_{i k}$ to the continuum equation. It represents an approximation to the exact solution $\Psi$,

$$
\psi_{i k}=\Psi_{i k}+E_{i k} \Delta^{p}+\ldots
$$

where $\Psi_{i k}=\Psi\left(\eta_{i}, \theta_{k}\right), \Delta$ is a typical grid scale, and $E_{i j}=\mathcal{O}(1)$. We expect that $p=2$, since a second order accurate approximation to equation (11) was used to generate $\psi_{i k}$. In section 2.2 a convergence test for the pure black hole solution confirmed that the continuum code is second order accurate.

Now consider the simplicial Regge solution $\psi_{i k}^{R}$, which we also expect to differ from the continuum solution by some amount dependent on the scale length $\Delta$. By this reasoning we see that the Regge solution should also differ from the numerical solution of the continuum equations by some small amount. If we assume that

$$
\psi_{i k}^{R}=\psi_{i k}+K_{i k} \Delta^{q}+\ldots,
$$

where $K_{i j}=\mathcal{O}(1)$, we can write

$$
\frac{\psi_{i k}^{R}-\psi_{i k}}{\psi_{i k}}=\frac{K_{i k}}{\Psi_{i k}} \Delta^{q}+\ldots
$$

where we have neglected only terms smaller than $\Delta^{q}$. Noting that the co-efficient of $\Delta^{q}$ scales as order unity, we define the "error" between the numerical continuum and Regge solutions as

$$
e_{N}=\frac{1}{N} \sum_{i=0}^{n_{\eta}} \sum_{k=0}^{n_{\theta}}\left|\frac{\psi_{i k}^{R}-\psi_{i k}}{\psi_{i k}}\right|
$$




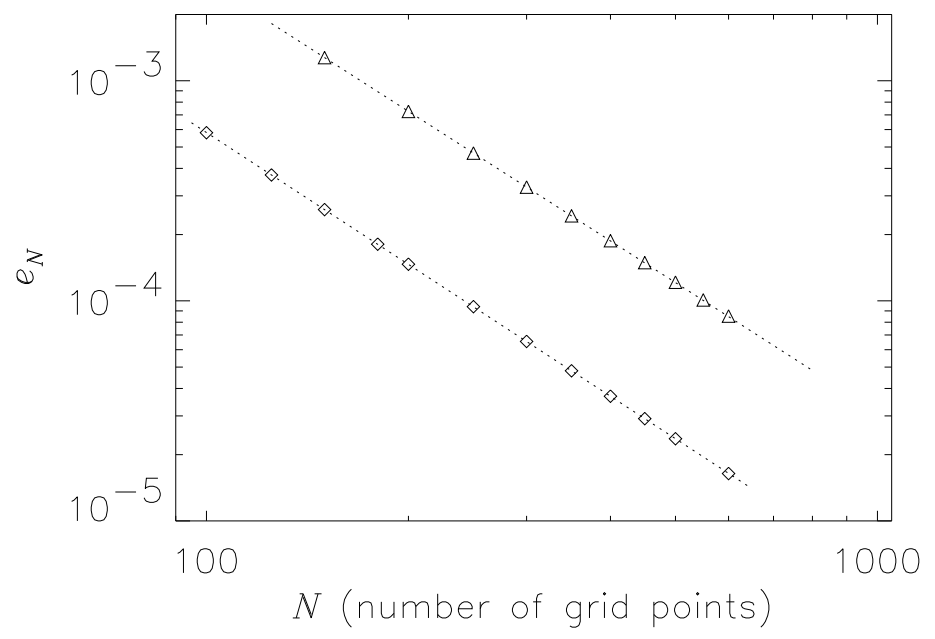

Figure 7. The averaged fractional difference $e_{N}$ between the simplicial and continuum solutions, shown as a function of the number of vertices (grid points). All calculations were performed on an $N \times N$ grid, for the pure Brill wave space with $a=10$ (triangles), and distorted black hole initial data with parameters $(1,2,1)$ (diamonds). In both cases, the fractional difference between the simplicial Regge and continuum solutions reduces as the second power of the grid spacing. From this we conclude that the simplicial Regge solution is a second order accurate approximation to the underlying continuum solution.

where $N=\left(n_{\eta}+1\right) \times\left(n_{\theta}+1\right)$. The error $e_{N}$ may be calculated directly from the two numerical solutions, and doing so for a variety of grid scales $\Delta$ yields an estimate of the convergence rate $q$.

Figure 7 shows the behaviour of the error $e_{N}$ as the number of grid points is increased (hence $\Delta$ decreased) for both the pure Brill wave and the distorted black hole initial data sets. The figure suggests a value of $q \approx 2$. That is, the difference between the simplicial Regge solution and the numerical solution $\psi_{i k}$ decreases as approximately the second power of the discretization scale. Since the numerical solution $\psi_{i k}$ itself differs the exact solution $\Psi$ by terms of order $\Delta^{2}$, we conclude that the Regge solution is a second order accurate approximation to $\Psi$, the underlying exact solution of the continuum equations.

\section{Discussion and further work}

We have successfully used Regge calculus to construct axisymmetric initial data at a moment of time symmetry. Brill wave and distorted black hole initial data sets were obtained, and shown to agree well with the corresponding continuum solutions.

The simplicial lattice - consisting of tetrahedra in three-dimensions - was found to yield solutions in excellent agreement with the continuum, and displayed none of the problems encountered with a prism-based Regge lattice. In particular, we observed that the simplicial Regge solution converged to the numerical solution of Einstein's equations as the second power of the lattice spacing. From this it was inferred that 
the simplicial Regge initial data is a second order accurate approximation to the exact solution.

Even for axisymmetric gravitational waves at a moment of time symmetry, we have found it necessary to abandon the prism-based lattice in favour of a simplicial approach. This indicates that the future development of Regge calculus as a competitive and robust alternative to standard numerical techniques will require the use of fully simplicial lattices.

Work is currently underway to evolve this data set, in $(2+1)$-dimensions using an axisymmetric adaptation of the Sorkin evolution scheme [3, 4 , 5], as well as a fully $(3+1)$-dimensional evolution.

\section{Acknowledgments}

The author wishes to thank Leo Brewin, Warner Miller and Daniel Holz for many stimulating discussions on this and related work. Financial support was provided by the Sir James McNeill Foundation at Monash University, the Center for Nonlinear Studies at Los Alamos, and the Theoretical Astrophysics group at Los Alamos through an LDRD grant.

\section{References}

[1] T. Regge, Nuovo Cimento , 19, 558-71 (1961).

[2] R. M. Williams and P. A. Tuckey, Class. Quantum Grav. , 9, 1409-22 (1992).

[3] R. D. Sorkin, Phys. Rev. , D12, 385-96 (1975).

[4] P. A. Tuckey, Class. Quantum Grav. , 10, L109-13 (1992).

[5] J. W. Barrett, M. Galassi, W. A. Miller, R. D. Sorkin, P. A. Tuckey and R. M. Williams, Int. J. Theor. Phys., 36, 815-39 (1997).

[6] A. P. Gentle and W. A. Miller, Class. Quantum Grav. , 15, 389-405 (1998).

[7] D. R. Brill, Ann. Phys., 7, 466-83 (1959).

[8] D. H. Bernstein, Ph.D Thesis, University of Illinois (1993)

[9] K. Eppley Phys. Rev. , D16, 1609-14 (1977).

[10] S. M. Miyama, Prog. Theor. Phys., 65, 894-909 (1981).

[11] D. E. Holz, W. A. Miller, M. Wakano and J. A. Wheeler, in Directions in General Relativity: Proceedings of the 1993 International Symposium, Maryland; Papers in Honour of Dieter Brill, eds. B. L. Hu and T. Jacobson (Cambridge Uni. Press, 1993).

[12] M. Alcubierre, S. Brandt, B. Brügmann, C. Gundlach, J. Masso, E. Seidel and P. Walker, gr-qc/9809004.

[13] K. D. Camarda, Ph.D Thesis, University of Illinois (1998)

[14] M. R. Dubal, Class. Quantum Grav. , 6, 141-55 (1989).

[15] A. P. Gentle, in Proc. First Australasian Conf. on Gen. Rel. Grav., ed. D. L. Wiltshire (Uni. Adelaide, 1996).

[16] C. W. Misner, K. S. Thorne and J.A. Wheeler, Gravitation, W.H. Freeman, San Francisco, 1973.

[17] J. A. Wheeler, in Relativity, Groups and Topology, ed. C. DeWitt and B. DeWitt (Blackie and Son Ltd., 1964)

[18] S. M. Lewis, Phys. Rev. , D25, 307-12 (1982).

[19] P. A. Collins and R. M. Williams, Phys. Rev. , D7, 965-71 (1973). 